\title{
The application of thromboelastography in risk stratification for selective thromboembolism prophylaxis after total joint arthroplasty in Chinese: a randomized controlled trial
}

\author{
Ziming Chen ${ }^{1,2}$, Yuanchen $\mathrm{Ma}^{1}$, Qingtian $\mathrm{Li}^{1}$, Zhantao Deng ${ }^{1}$, Qiujian Zheng ${ }^{1}$ \\ ${ }^{1}$ Department of Orthopedics, Guangdong Provincial People's Hospital, Guangdong Academy of Medical Sciences, Guangzhou, China; ${ }^{2}$ Centre for \\ Orthopaedic Translational Research, Medical School, University of Western Australia, Nedlands, Australia \\ Contributions: (I) Conception and design: Z Chen, Y Ma, Q Li, Q Zheng; (II) Administrative support: Q Zheng; (III) Provision of study materials or \\ patients: Q Li, Y Ma, Q Zheng; (IV) Collection and assembly of data: Z Chen, Q Li, Z Deng; (V) Data analysis and interpretation: Z Chen, Y Ma, Z \\ Deng; (VI) Manuscript writing: All authors; (VII) Final approval of manuscript: All authors. \\ Correspondence to: Zhantao Deng, PhD; Qiujian Zheng, MD. Department of Orthopedics, Guangdong Provincial People's Hospital, Guangdong \\ Academy of Medical Sciences, Guangzhou 510080, China. Email: dengzhantao_med@163.com; ZQJzqj666@yeah.net.
}

\begin{abstract}
Background: As Asian populations, Chinese have a lower rate of high-risk gene mutations in venous thrombosis. Therefore, individual patient risk assessment, rather than a "blanket policy", is considered the best thromboembolism prophylaxis for Chinese. The purpose of this study was to evaluate the effectiveness and safety of selective thromboembolism prophylaxis compared with conventional thromboembolism prophylaxis by risk stratification with thromboelastography (TEG) after joint arthroplasty in Chinese.

Methods: Between August 2016 to August 2017, Chinese patients who underwent hip or knee arthroplasty were randomly divided into a selective anticoagulation group (SAG) and a conventional anticoagulation group (CAG). Participants and outcome assessors were blinded. In the SAG, an anticoagulant was used when TEG indicated hypercoagulability; while in the CAG, an anticoagulant was regularly used until one month after surgery. Outcome evaluation included effectiveness (defined as the incidence of VTE), and safety (defined as the incidence of bleeding events, poor wound healing, blood loss, and infection).

Results: A total of 197 patients (79 in the SAG and 118 in the CAG) were included in the study. There was 1 case of deep vein thrombosis (DVT) in the SAG and 2 cases of DVT in the CAG, but there was no significant difference between the two groups. Hidden blood loss in the SAG was $707.4 \pm 539.8 \mathrm{~mL}$ and hidden blood loss in the CAG was $617 \pm 565.0 \mathrm{~mL}$, respectively $(\mathrm{P}>0.05)$. No significant difference was observed in perioperative blood loss between the SAG and the CAG $(1,024.9 \pm 597.9$ and $1,139.3 \pm 620.9 \mathrm{~mL}$, respectively). Volume of blood transfusion was $92.4 \pm 270.2 \mathrm{~mL}$ in the $\mathrm{SAG}$ and $224.6 \pm 416.3 \mathrm{~mL}$ in the CAG, respectively, while rate of transfusion was $13.9 \%$ in the SAG and $33.9 \%$ in the CAG, respectively, which were significantly different between the two groups $(\mathrm{P}<0.05)$.

Conclusions: In Chinese patients who underwent hip or knee arthroplasty, the efficacy of selective anticoagulation using TEG in risk stratification was comparable to that of conventional anticoagulation. Furthermore, the safety of selective anticoagulant prophylaxis was superior to that of conventional anticoagulant prophylaxis.
\end{abstract}

Keywords: Arthroplasty; deep vein thrombosis; thromboelastogram

Submitted Oct 17, 2019. Accepted for publication Jun 29, 2020.

doi: 10.21037/apm-19-385

View this article at: http://dx.doi.org/10.21037/apm-19-385

(c) Annals of Palliative Medicine. All rights reserved. 


\section{Introduction}

Venous thromboembolism (VTE), including asymptomatic and symptomatic deep vein thrombosis (DVT) and pulmonary thromboembolism (PE), is a life-threatening complication after total hip arthroplasty (THA) and total knee arthroplasty (TKA). However, the VTE prophylaxis remains a controversial topic in total joint arthroplasty. Different incidences of VTE have been reported in various studies. Zhou et al. found that Asian populations have a lower rate of high-risk gene mutations (1). Although many studies have shown that the incidence of DVT is lower in Asian patients than in the Western population (2-4), some studies from China and southeast Asia have still shown significant incidence of DVT in high-risk orthopaedic patients $(5-7)$.

In terms of prophylaxis protocol, American Academy of Orthopaedic Surgeons (AAOS) and the American College of Chest Physicians (ACCP) typically recommended the routine use of chemotherapeutic agents $(8,9)$. For Chinese, the Chinese Medical Association advised a 35-day routine chemoprophylaxis after THA and at least a 10-14 day routine chemoprophylaxis after TKA (10). However, W.G. Hamilton et al. simply used a mechanical program without any chemotherapeutic agent, and finally, they found that it was safe and effective (11). But there is a lack of sufficient researches that proves whether it is safe to exclude a chemoprophylactic agent.

Actually, all of these guidelines provided some stratification for patients on the basis of the risk of clotting as well as that of bleeding. Although most of the patients are recommended to receive routine chemoprophylaxis, it is not recommended to use anticoagulants in some patients who are at high risk of bleeding (8-10). Moreover, some researches also used risk stratification to identify the types of patients requiring the chemotherapeutic agent, and then they provided a more individualized and precise protocol for their patients (11). However, the stratification used in the research ranged from the use of risk assessment tables to scoring systems, with an occasional use of a long list of risk factors (12-14), which was delayed. Few of stratification methods were specially built for patients who underwent THA or TKA. Currently, there is a detection technique called thromboelastography (TEG), which is a real-time tool to observe the whole process of blood coagulation and is clinically valuable in the evaluation of whole-blood hemostasis (15).

In this study, we designed selective thromboembolism chemoprophylaxis after THA or TKA in Chinese. Risk stratification was performed by TEG. The purpose of this study was to evaluate the effectiveness and safety of selective thromboembolism prophylaxis compared with conventional thromboembolism prophylaxis by risk stratification with TEG after joint arthroplasty in Chinese. We present the following article in accordance with the CONSORT reporting checklist (available at http://dx.doi.org/10.21037/ apm-19-385).

\section{Methods}

This study was performed in accordance with the ethical standards laid down in the Helsinki Declaration as revised in 2013. All of the patients gave their informed consent prior to their inclusion in the study. The study was approved by institutional human ethics committee of Guangdong Provincial People's Hospital (No. 2016-46). The trial protocol was submitted to ClinicalTrials.gov, and the ClinicalTrials.gov Identifier number is NCT03804697.

\section{Source of data and participants}

This was a single-center, participant- and outcome assessorblinded, randomized controlled trial. Asian patients who underwent hip or knee arthroplasty in the Department of Orthopedics (name of institution blinded) from August 2016 to August 2017 were included. All of the cases were categorized into the same treatment group, with 6 surgeons.

\section{Inclusion criteria and exclusion criteria}

Inclusion criteria were as follows: patients aged $>18$ years and patients who underwent a unilateral or bilateral primary TKA/THA.

Exclusion criteria were as follows: patients who had a history of DVT or PE (these patients would receive lowmolecular-weight heparin and warfarin postoperatively), patients who were placed on chronic warfarin therapy, patients whose body mass index (BMI) was $\geq 40 \mathrm{~kg} / \mathrm{m}^{2}$, patients whose liver and kidney function was too low to use an oral anticoagulant or low molecular weight heparin, and patients who were scheduled for other surgeries within 3 months.

\section{Study design}

The participants underwent a baseline assessment after they 
were determined to be eligible for the study.

Patients were randomly allocated to the selective anticoagulation group (SAG) or the conventional anticoagulation group (CAG). We used coin tossing to decide grouping. An author generated the random allocation sequence, enrolled participants, and assigned participants to interventions.

All of the patients and surgeons were blinded to the treatment options. Only one resident was informed about the group assignment and he/she prescribed an anticoagulant for patients if needed.

TEG procedure were performed 1 day before the surgery, 1 day after the surgery, 3 days after the surgery, and 5 days after the surgery, and the definition of hypercoagulable state used in this study was previously described by Kaufmann et al. as the presence of at least two of the following: shortened $R$ time, increased $\alpha$ angle, and increased MA (16).

DVT was diagnosed by ultrasound, while the volume of blood loss was calculated by the Nadler Formula as followed: patient's blood volume $(\mathrm{PBV})=\mathrm{k}_{1}$ * height $(\mathrm{m})^{3}$ $+\mathrm{k}_{2}{ }^{*}$ weight $(\mathrm{kg})+\mathrm{k}_{3}$. For male patients, $\mathrm{K}_{1}=0.3669, \mathrm{~K}_{2}=$ $0.03219, \mathrm{~K}_{3}=0.6041$, while for female patients, $\mathrm{K}_{1}=0.3561$, $\mathrm{K}_{2}=0.033083, \mathrm{~K}_{3}=0.1833$. Total erythrocyte loss = PBV * (perioperative HCT - HCT 3 days after the surgery). Total blood loss = Total erythrocyte loss $/$ preoperative HCT. Perioperative blood loss $=$ total blood loss + blood transfusion. Hidden blood loss = perioperative blood loss - intraoperative blood loss - postoperative drainage. TEG was tested using an analyzer (5000 Thrombelastograph Hemostasis Analyzer System, Haemoscope, USA).

As shown in Figure 1, in the SAG, an anticoagulant was used once TEG indicated a hypercoagulable state; while in the CAG, an anticoagulant was routinely used until one month after surgery.

The dosage regimen was as follows: hypodermic injection of $0.4 \mathrm{ml}$ enoxaparin (0.6 mL: 6000 A*aIU, Clexane $^{\circledR}$, Sanofi Winthrop Industrie, France) per day for 5 days, followed by oral administration of $10 \mathrm{mg}$ rivaroxaban (Xarelto ${ }^{\circledR}$, Bayer Pharma AG, Germany) per day until one month after the surgery.

\section{Clinical data}

Preoperative data included patients' basic information (name, gender, BMI, age, diagnosis, and date of surgery), complete blood count (CBC), and TEG results 1 day before the surgery. Intraoperative data included volume of intraoperative blood loss and volume of intraoperative blood transfusion. Postoperative data included CBC, postoperative volume of drainage, blood transfusion, blood loss, TEG results (recorded 1 day after the surgery, 3 days after the surgery, and 5 days after the surgery), and the incidence rate of VTE (recorded during hospitalization, 1 month after the surgery, 3 months after the surgery, and 6 months after surgery). Anticoagulation-related complications, such as ecchymosis that was larger than $25 \mathrm{~cm}^{2}$ and beyond $10 \mathrm{~cm}$ of incision and postoperative infection that included superficial infection and deep infection, were also recorded. In the present study, the incidence rate of VTE is the primary outcome.

Previous publications were used to determine the power of both groups $(17,18)$. At least 50 patients per group are necessary to measure the difference with a bilateral alpha of 0.05 and a power of $80 \%$. However, there was no accurate incidence rate of DVT in Asian populations. Finally, we included a minimum of 79 patients in each group.

\section{Statistical analysis}

T-test was used to compare the mean of independent samples between the two groups. Variables are described as the mean and the standard deviation of the mean. The mean comparison between multiple independent samples was analyzed by ANOVA. Mann-Whitney Test was used to compare the data of abnormal distributions between the two groups, such as the volume of transfusion in this study. Chisquare test was used to compare categorical variables, such as complication rate and the incidence of complications.

SPSS 21.0 statistical analysis software (SPSS, Chicago, IL, USA) was used for statistical analysis. The level of significance for all tests was defined as a $\mathrm{P}$ value of less than 0.05 .

\section{Results}

A total of 197 patients with an average age of $65 \pm 12$ years from August 2016 to August 2017 were included in the study. Of these 197 patients, 79 patients (34 underwent hip surgery and 45 underwent knee surgery) were allocated to the SAG and 118 patients (51 underwent hip surgery and 67 underwent knee surgery) were allocated to the CAG. Finally, 28 patients in the SAG received chemoprophylaxis and 51 patients in the SAG did not receive chemoprophylaxis (Figure 1). No data are missing in this study.

The baseline data of the patients is shown in Table 1 . 


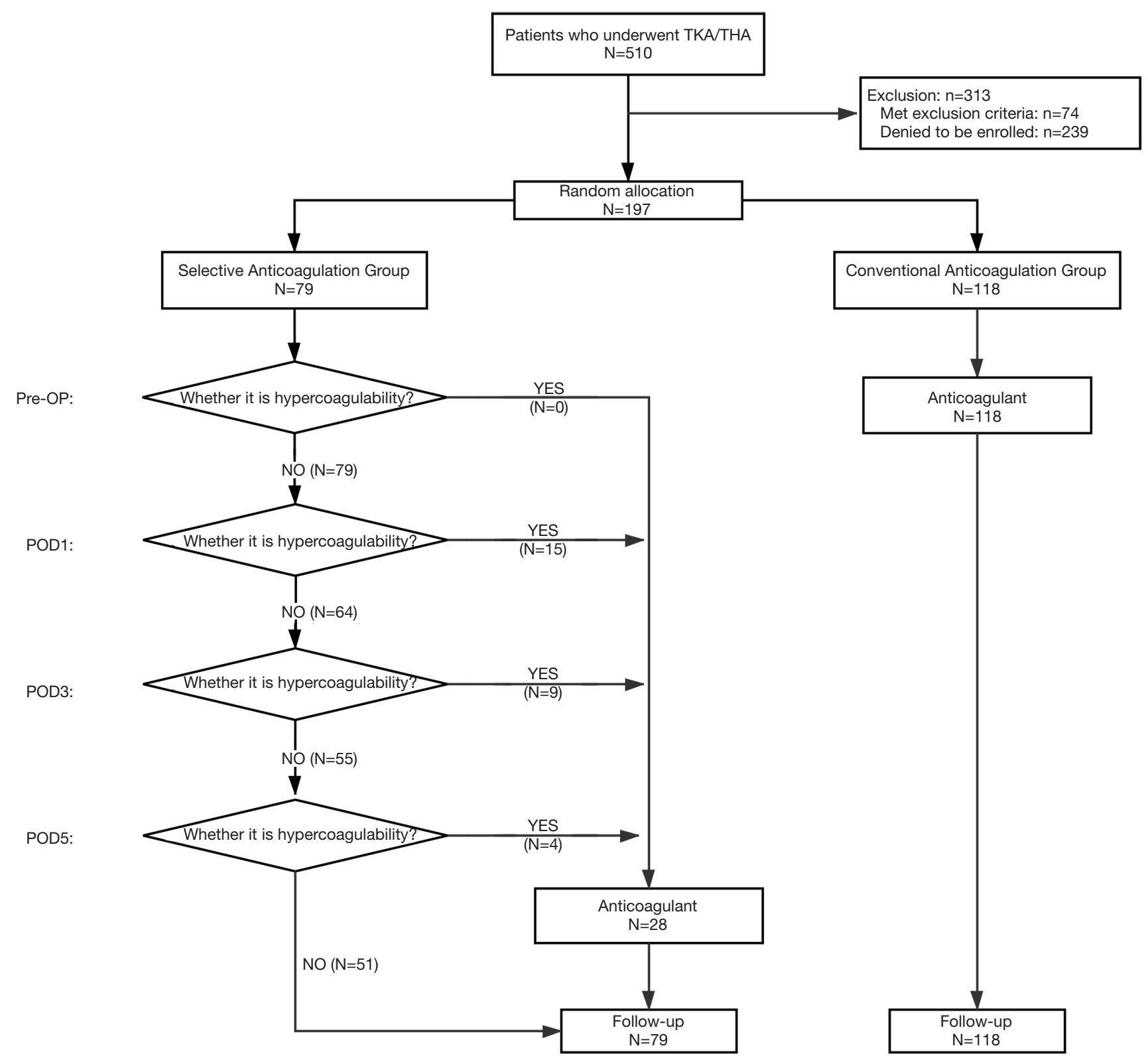

Figure 1 Flow chart of the study design. Pre-OP, preoperative day; POD1, postoperative day 1; POD 3, postoperative day 3; POD 5, postoperative day 5 .

There was no significant difference between the two groups in terms of baseline characteristics, indicating that the samples in the two groups were randomly distributed and the two groups of samples were comparable.

\section{Results for effectiveness}

The incidence rate of DVT was the primary outcome of this study, which indicated the effectiveness of the protocol. The results showed that there was no significant difference in the incidence rate of DVT between the two groups during hospitalization, 1 month after the operation, 3 months after the operation, and 6 months after the operation, thus suggesting that the clinical efficacy of selective anticoagulation was similar to that of conventional anticoagulation (Table 2). Also, PE was not occurred in the 
Table 1 Baseline characteristics

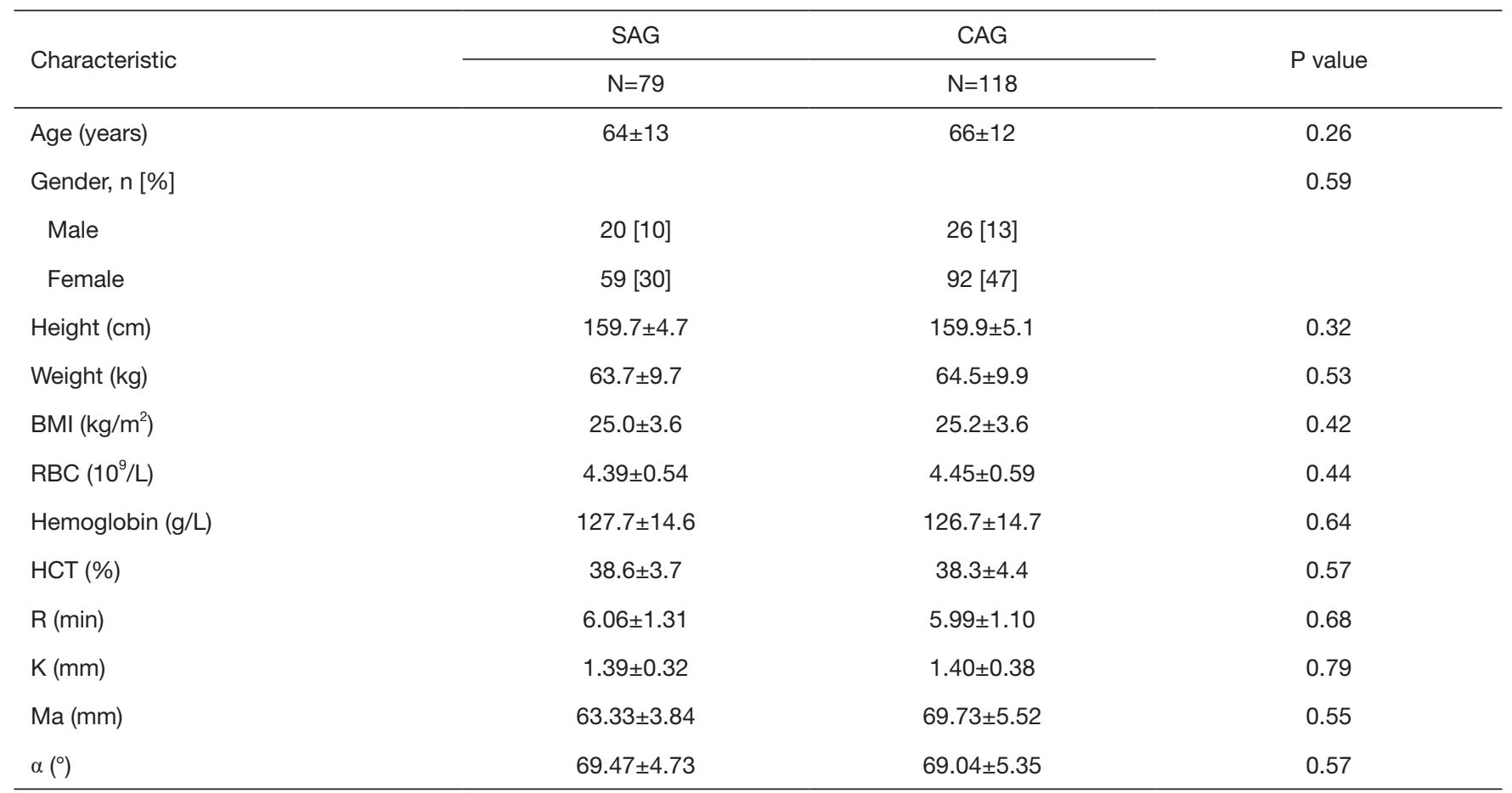

SAG, selective anticoagulation group; CAG, conventional anticoagulation group; N, number of cases; RBC, red blood cell; BMI, body mass index; HCT, hematocrit

Table 2 The Incidence of DVT in the two groups

\begin{tabular}{lccc}
\hline DVT & SAG $(\mathrm{N}=79), \mathrm{n}(\%)$ & $\mathrm{CAG}(\mathrm{N}=118), \mathrm{n}(\%)$ & $\mathrm{P}$ value \\
\hline Hospitalization & 0 & $2(1.7)$ & 0.245 \\
1 month after the operation & $1(1.2)$ & $2(1.7)$ & 0.81 \\
3 months after the operation & $1(1.2)$ & $2(1.7)$ & 0.81 \\
6 months after the operation & $1(1.2)$ & $2(1.7)$ & 0.81 \\
\hline
\end{tabular}

DVT, deep vein thrombosis; N, number of cases; SAG, selective anticoagulation group; CAG, conventional anticoagulation group.

two groups.

\section{Results for safety}

As illustrated in Figure 2, the levels of perioperative hematocrit (HCT), hemoglobin (HGB), and RBCs in the two groups decreased within 3 days after the operation, reached the lowest point on the third day after the operation, and then they increased. There was no significant difference between the two groups at different time points, thus suggesting that the loss of blood volume after the operation was similar between the two groups.

There was no significant difference between the two groups in terms of hidden blood loss, perioperative blood loss, and total blood loss (Figure 3).

Blood transfusion volume and transfusion rate in the SAG were lower than those in the CAG, and there was a significant difference between the two groups $(\mathrm{P}=0.002)$. This suggested that selective anticoagulation could reduce the volume of transfusion (92.4 vs. $224.6 \mathrm{~mL}$ ) and the transfusion rate (13.9\% vs. $33.9 \%)$, thus the safety of selective anticoagulation was better than that of conventional anticoagulation (Table 3).

There was no significant difference in the infection rate between the two groups (Table 4). In this study, 3 cases of ecchymosis occurred in the SAG and 13 cases of ecchymosis 


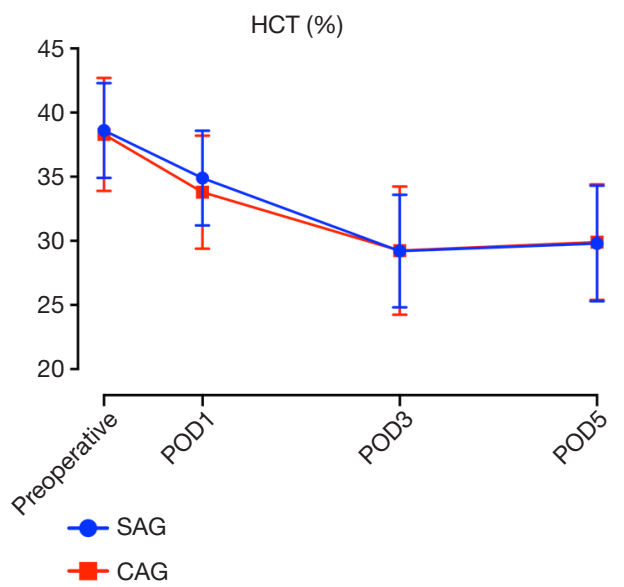

$\mathrm{HGB}(\mathrm{g} / \mathrm{L})$

$\operatorname{RBC}\left(10^{9} / \mathrm{L}\right)$

Figure 2 Perioperative HCT, HGB and RBC. HCT, hematocrit; HGB, hemoglobin; RBC, red blood cell; SAG, selective anticoagulation group; CAG, conventional anticoagulation group.

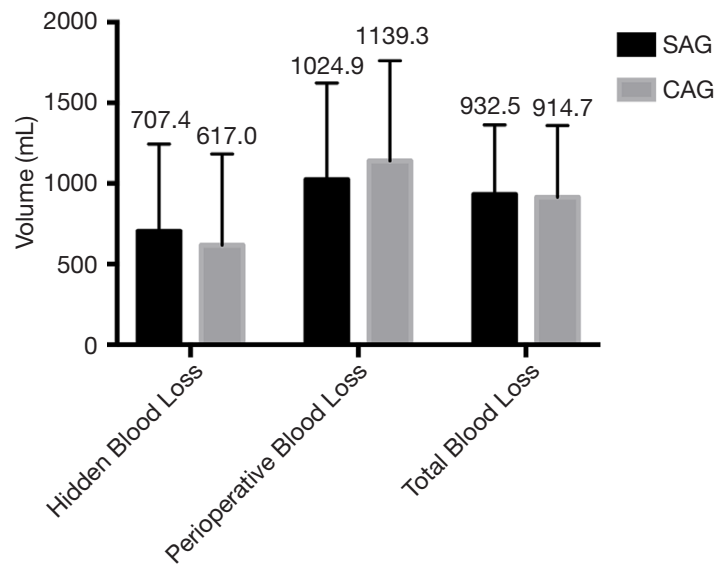

Figure 3 Blood loss in SAG and CAG. SAG, selective anticoagulation group; CAG, conventional anticoagulation group.

occurred in the CAG. However, there was no significant difference in the ecchymosis rate between the two groups, which indicated that use of a selective anticoagulant did not increase the risk of bleeding (Table 5).

\section{Discussion}

In our study, the use of a selective anticoagulant did not increase the incidence rate of DVT compared with the use of a routine anticoagulant, which means the efficacy of selective anticoagulation is comparable to that of conventional anticoagulation. Moreover, there was no significant difference in the postoperative infection rate and ecchymosis incidence, but the volume of blood transfusion and blood transfusion rate in the SAG were lower than those in the CAG, which means that the safety of selective anticoagulant prophylaxis is superior to that of conventional anticoagulant prophylaxis.

It is now known that the type of thromboprophylaxis is not uniformly indicated for an individual patient because a uniform protocol makes it difficult to maintain the balance between effectiveness, contraindications, side effects, and cost $(19,20)$. For these reasons, currently some approaches are used for the stratification of patients into defined risk categories, such as risk assessment models and some scoring systems. However, the stratification is always based on patients' preoperative characteristics, such as age, surgical procedure, history of VTE, presence of malignancy, and thrombophilia, which means that it is cannot reflect the real-time situation of thrombophilia $(9,12,21)$. TEG provides a direct overview of the clotting process, from initial thrombin generation to formation of fibrin strands and fibrinolysis, which means that it is real time and can instantly reflect thrombophilia $(16,22)$. Moreover, it can sensitively identify patients with postinjury hypercoagulability (23-25). However, the use of TEG to assess the risk of VTE and then to guide the regimen for pharmacological prophylaxis has not yet been described. In our study, we found that risk stratification by TEG to selectively prevent VTE was effective.

On the one hand, although many studies have provided the clinical practice guidelines for VTE prophylaxis, there is no standard prophylaxis guideline for VTE prevention after arthroplasty specifically (8-10). Furthermore, in these guidelines, patients who underwent THA and TKA 
Table 3 Blood transfusion volume and transfusion rate in the two groups

\begin{tabular}{lccc}
\hline Blood Transfusion & SAG & CAG & P value \\
\hline Rate of transfusion $(\%)$ & $13.9(\mathrm{~N}=11)$ & $33.9(\mathrm{~N}=40)$ & 0.002 \\
Volume of transfusion $(\mathrm{mL})$ & $92.4 \pm 270.2$ & $224.6 \pm 416.3$ & 0.002 \\
\hline
\end{tabular}

SAG, selective anticoagulation group; CAG, conventional anticoagulation group; N, number of cases that received blood transfusion.

Table 4 Infection in the two groups

\begin{tabular}{|c|c|c|c|c|c|c|}
\hline Infection & \multicolumn{3}{|c|}{ Superficial infection, n (\%) } & \multicolumn{3}{|c|}{ Deep infection, n (\%) } \\
\hline Hospitalization & 0 & 0 & & 0 & 0 & \\
\hline 1 month after surgery & & $1(1.3)$ & 0.412 & $1(1.3)$ & $1(1.3)$ & 0.774 \\
\hline 3 months after surgery & 0 & $1(1.3)$ & 0.412 & $1(1.3)$ & $1(1.3)$ & 0.774 \\
\hline
\end{tabular}

SAG, selective anticoagulation group; CAG, conventional anticoagulation group; N, number of cases.

Table 5 Ecchymosis incidence in the two groups

\begin{tabular}{lccc}
\hline Ecchymosis & SAG & CAG & P value \\
\hline $\mathrm{N}$ & 3 & 13 & \\
Rate $(\%)$ & 3.8 & 11 & 0.069 \\
\hline
\end{tabular}

SAG, selective anticoagulation group; CAG, conventional anticoagulation group; $\mathrm{N}$, number of cases.

were at high risk of DVT, which means that these patients should accept uniform chemoprophylaxis. Thus, a socalled "carpet" approach for uniform prophylaxis has been proposed (26-28).

On the other hand, some researchers used an extreme method that did not include any chemoprophylaxis for Asian patients $(29,30)$. The underlying reason is as follows: in high-risk situations in Western countries, the incidence of VTE is generally thought to be low in Asians $(3,4,31)$. A retrospective study by Park et al. showed that in 1933 patients after knee arthroplasty, the incidence of symptomatic V'TE after simple mechanical thromboembolic prophylaxis was $0.9 \%$ (31). A recent epidemiological study based on 306,912 cases in Korea also showed the incidence of VTE was low without chemoprophylaxis (TKA, 1.14\%; THA, $1.43 \%$ ) (3). However, the results of some studies were not in agreement with those of that study (32). These studies found that the incidence of VTE in Asian patients who underwent major orthopedic surgery, such as hip fracture surgery, without chemoprophylaxis was 50.0\% (33), and the incidence of VTE ranged from $9.8 \%$ to $64.3 \%$ after THA (33-35), and from $11 \%$ to $52.8 \%$ after TKA $(17,36)$. Furthermore, the incidence of postoperative PE is not negligible in Asian patients who underwent surgery: it was about $1.4 \%$ (37). Thus, Asian patients are at risk of developing VTE if they do not receive chemoprophylaxis.

Studies have shown that reasonable use of anticoagulants can effectively reduce the incidence of VTE after hip and knee arthroplasty (38-40). However, inappropriate use of anticoagulants can increase the incidence of adverse events, such as infection, poor wound healing, and increased blood loss $(27,41,42)$. Clinicians paid attention to the clinical preventive effect of drugs and ignored their adverse reactions. We now know that before deciding to use anticoagulants, the benefits of thrombosis reduction and the risks of drug-related infections and bleeding should be fully assessed. In other words, a safe and reliable screening method is very important. Our study proved that selective anticoagulation using TEG for risk stratification can reduce the volume of transfusion and transfusion rate, and this finding indicates that selective anticoagulation is safer than traditional anticoagulation.

There are some limitations in this study. First, this study was a single-center, randomized controlled study with a small sample size. The results of this study need to be strengthened and verified further by a multi-center study. Second, because of the interaction between rivaroxaban and antihypertensive drugs, enoxaparin was chosen as a preventive drug during hospitalization, and rivaroxaban 
was chosen after discharge because it is convenient to carry and use the drug. There are differences in efficacy among different patients because rivaroxaban can be affected by antihypertensive drugs, which may cause bias in the incidence of VTE after discharge. However, because both groups of patients used the same anticoagulation regimen, this difference can be ignored. Finally, guidelines suggest that 1 month post-surgery is a high-risk period for thrombosis, but this study only tested the patients who underwent TEG 5 days after surgery. The main consideration is that the TEG test should be carried out in a hospital, but patients are usually discharged within 5 days after the operation. If patients have to continue TEG examination up to one month after the operation, their financial and time burden will increase and the feasibility of the scheme will reduce. Therefore, after 5 days, patients should pay close attention to the condition of their lower limbs. If symptoms related to VTE, such as pain and swelling occur, patients should return to the hospital for reexamination.

\section{Conclusions}

In Chinese patients who underwent hip or knee arthroplasty, the efficacy of selective anticoagulation was comparable to that of conventional anticoagulation. Furthermore, the safety of selective anticoagulant prophylaxis was superior to that of conventional anticoagulant prophylaxis. To sum up, this regimen is easy to administer and cost effective, and it would have a lower risk of bleeding than the protocols recommended by the guideline.

\section{Acknowledgments}

We would like to thank Letpub Language Editing Service (https://www.letpub.com) for checking the wordings of the present study. And we would like to thank Dr. Junfeng Chen for collecting some data and his statistical assistance.

Funding: This study was supported by Provincial Science and Technology Major Project of Guangdong (2015B020225007), the National Natural Science Foundation of China Youth Science Foundation (81802222), the Natural Science Foundation of Guangdong Province (2018A030310694, 2020A1515010268), the Foundation of Traditional Chinese Medicine of Guangdong Province (20191004), the Scientific Foundation of Guangdong Provincial People's Hospital (2017bp01), the Outstanding
Young Talents Foundation of Guangdong Provincial People's Hospital (KJ012019091), the Program of Science and Technology of Guangzhou (201904010424), and the Guangdong Medical Science and Technology Research Foundation (2018114214430383).

\section{Footnote}

Reporting Checklist: The authors have completed the CONSORT Reporting Checklist. Available at http://dx.doi. org/10.21037/apm-19-385

Data Sharing Statement: Available at http://dx.doi. org/10.21037/apm-19-385

Conflicts of Interest: All authors have completed the ICMJE uniform disclosure form (available at http://dx.doi. org/10.21037/apm-19-385). All authors report grants from Natural Science Foundation of Guangdong, grants from National Natural Science Foundation of China, grants from Department of science and technology of Guangdong Province, grants from Guangdong Provincial Bureau of traditional Chinese Medicine, grants from Bureau of Science and Technology of Guangzhou, grants from Health Commission of Guangdong Province, during the conduct of the study.

Ethical Statement: The authors are accountable for all aspects of the work in ensuring that questions related to the accuracy or integrity of any part of the work are appropriately investigated and resolved. This study was performed in accordance with the ethical standards laid down in the Helsinki Declaration as revised in 2013. All of the patients gave their informed consent prior to their inclusion in the study. The study was approved by institutional human ethics committee of Guangdong Provincial People's Hospital (No. 2016-46).

Open Access Statement: This is an Open Access article distributed in accordance with the Creative Commons Attribution-NonCommercial-NoDerivs 4.0 International License (CC BY-NC-ND 4.0), which permits the noncommercial replication and distribution of the article with the strict proviso that no changes or edits are made and the original work is properly cited (including links to both the formal publication through the relevant DOI and the license). See: https://creativecommons.org/licenses/by-nc-nd/4.0/. 


\section{References}

1. Zhou X, Qian W, Li J, et al. Who are at risk for thromboembolism after arthroplasty? A systematic review and meta-analysis. Thromb Res 2013;132:531-6.

2. Bagaria V, Modi N, Panghate A, et al. Incidence and risk factors for development of venous thromboembolism in Indian patients undergoing major orthopaedic surgery: results of a prospective study. Postgrad Med J 2006;82:136-9.

3. Yhim HY, Lee J, Lee JY, et al. Pharmacological thromboprophylaxis and its impact on venous thromboembolism following total knee and hip arthroplasty in Korea: A nationwide population-based study. PLoS One 2017;12:e0178214.

4. Yeo DX, Junnarkar S, Balasubramaniam S, et al. Incidence of venous thromboembolism and its pharmacological prophylaxis in Asian general surgery patients: a systematic review. World J Surg 2015;39:150-7.

5. Wang CJ, Wang JW, Weng LH, et al. Prevention of deep-vein thrombosis after total knee arthroplasty in Asian patients. Comparison of low-molecular-weight heparin and indomethacin. J Bone Joint Surg Am 2004;86-a:136-40.

6. Piovella F, Wang CJ, Lu H, et al. Deep-vein thrombosis rates after major orthopedic surgery in Asia. An epidemiological study based on postoperative screening with centrally adjudicated bilateral venography. J Thromb Haemost 2005;3:2664-70.

7. Parameswaran A, Krishnamoorthy VP, Oommen AT, et al. Is pre-operative assessment of coagulation profile with Thrombelastography (TEG) useful in predicting venous thromboembolism (VTE) following orthopaedic surgery? J Clin Orthop Trauma 2016;7:225-9.

8. Johanson NA, Lachiewicz PF, Lieberman JR, et al. American academy of orthopaedic surgeons clinical practice guideline on. Prevention of symptomatic pulmonary embolism in patients undergoing total hip or knee arthroplasty. J Bone Joint Surg Am 2009;91:1756-7.

9. Geerts WH, Bergqvist D, Pineo GF, et al. Prevention of venous thromboembolism: American College of Chest Physicians Evidence-Based Clinical Practice Guidelines (8th Edition). Chest 2008;133:381s-453s.

10. Qiu G. Chinese Guidelines for prevention of venous thromboembolism after major orthopedic surgery. Chin J Joint Surg (Electronic Edition) 2016;36:65-71.

11. Hamilton WG, Reeves JD, Fricka KB, et al. Mechanical thromboembolic prophylaxis with risk stratification in total knee arthroplasty. J Arthroplasty 2015;30:43-5.

12. Nicolaides AN, Fareed J, Kakkar AK, et al. Prevention and treatment of venous thromboembolism--International Consensus Statement. Int Angiol 2013;32:111-260.

13. Liew NC, Alemany GV, Angchaisuksiri P, et al. Asian venous thromboembolism guidelines: updated recommendations for the prevention of venous thromboembolism. Int Angiol 2017;36:1-20.

14. Pannucci CJ, Bailey SH, Dreszer G, et al. Validation of the Caprini risk assessment model in plastic and reconstructive surgery patients. J Am Coll Surg 2011;212:105-12.

15. Mou Q, Zhou Q, Liu S. Blood clot parameters: Prejudgment of fibrinolysis in thromboelastography. Clin Chim Acta 2018;479:94-7.

16. Kaufmann CR, Dwyer KM, Crews JD, et al. Usefulness of thrombelastography in assessment of trauma patient coagulation. J Trauma 1997;42:716-20; discussion 20-2.

17. Kim YH, Yoo JH, Kim JS. Factors leading to decreased rates of deep vein thrombosis and pulmonary embolism after total knee arthroplasty. J Arthroplasty 2007;22:974-80.

18. Raphael IJ, Tischler EH, Huang R, et al. Aspirin: an alternative for pulmonary embolism prophylaxis after arthroplasty? Clin Orthop Relat Res 2014;472:482-8.

19. Berger RE, Pai M, Rajasekhar A. Thromboprophylaxis after Knee Arthroscopy. N Engl J Med 2017;376:580-3.

20. Runner RP, Gottschalk MB, Staley CA, et al. Utilization Patterns, Efficacy, and Complications of Venous Thromboembolism Prophylaxis Strategies in Primary Hip and Knee Arthroplasty as Reported by American Board of Orthopedic Surgery Part II Candidates. J Arthroplasty 2019;34:729-34.

21. Sultan AA, West J, Grainge MJ, et al. Development and validation of risk prediction model for venous thromboembolism in postpartum women: multinational cohort study. BMJ 2016;355:i6253.

22. Konstantinidi A, Sokou R, Parastatidou S, et al. Clinical Application of Thromboelastography/ Thromboelastometry (TEG/TEM) in the Neonatal Population: A Narrative Review. Semin Thromb Hemost 2019;45:449-57.

23. Salinas D. Viscoelastic Studies: Effective Tools for Trauma and Surgical Resuscitation Efforts. Aorn j 2017;105:370-83.

24. Coleman JR, Kay AB, Moore EE, et al. It's sooner than you think: Blunt solid organ injury patients are already hypercoagulable upon hospital admission - Results of a bi-institutional, prospective study. Am J Surg 
2019;218:1065-73.

25. Sumislawski JJ, Kornblith LZ, Conroy AS, et al. Dynamic coagulability after injury: Is delaying venous thromboembolism chemoprophylaxis worth the wait? J Trauma Acute Care Surg 2018;85:907-14.

26. Howard A, Zaccagnini D, Ellis M, et al. Randomized clinical trial of low molecular weight heparin with thighlength or knee-length antiembolism stockings for patients undergoing surgery. Br J Surg 2004;91:842-7.

27. Sun G, Wu J, Wang Q, et al. Factor Xa Inhibitors and Direct Thrombin Inhibitors Versus Low-MolecularWeight Heparin for Thromboprophylaxis After Total Hip or Total Knee Arthroplasty: A Systematic Review and Meta-Analysis. J Arthroplasty 2019;34:789-800.e6.

28. Hu KZ, Sun HY, Sui C. Effects of five treatment regimens on blood loss and blood transfusion in total knee arthroplasty: a preliminary study in China Int J Clin Pharmacol Ther 2017;55:433-41.

29. Yokote R, Matsubara M, Hirasawa N, et al. Is routine chemical thromboprophylaxis after total hip replacement really necessary in a Japanese population? J Bone Joint Surg Br 2011;93:251-6.

30. Moon KH, Kang JS, Youn YH. Is the Oral Chemical Prophylaxis Necessary for the Prevention of Venous Thromboembolism after Joint Arthroplasty in a Korean Population? Hip Pelvis 2019;31:150-7.

31. Park YG, Ha CW, Lee SS, et al. Incidence and Fate of "Symptomatic" Venous Thromboembolism After Knee Arthroplasty Without Pharmacologic Prophylaxis in an Asian Population. J Arthroplasty 2016;31:1072-7.

32. Leizorovicz A, Turpie AG, Cohen AT, et al. Epidemiology of venous thromboembolism in Asian patients undergoing major orthopedic surgery without thromboprophylaxis. The SMART study. J Thromb Haemost 2005;3:28-34.

33. Dhillon KS, Askander A, Doraismay S. Postoperative deep-vein thrombosis in Asian patients is not a rarity: a prospective study of 88 patients with no prophylaxis. J Bone Joint Surg Br 1996;78:427-30.

34. Yoo MC, Kang CS, Kim YH, et al. A prospective

Cite this article as: Chen Z, Ma Y, Li Q, Deng Z, Zheng Q. The application of thromboelastography in risk stratification for selective thromboembolism prophylaxis after total joint arthroplasty in Chinese: a randomized controlled trial. Ann Palliat Med 2020;9(5):2498-2507. doi: 10.21037/apm-19-385 randomized study on the use of nadroparin calcium in the prophylaxis of thromboembolism in Korean patients undergoing elective total hip replacement. Int Orthop 1997;21:399-402.

35. Kim YH, Choi IY, Park MR, et al. Deep vein thrombosis after uncemented total hip replacement. Bull Hosp Jt Dis 1997;56:133-9.

36. Fuji T, Nakamura M, Takeuchi M. Darexaban for the prevention of venous thromboembolism in Asian patients undergoing orthopedic surgery: results from 2 randomized, placebo-controlled, double-blind studies. Clin Appl Thromb Hemost 2014;20:199-211.

37. Leizorovicz A, Turpie AGG, Cohen AT, et al. Epidemiology of Post-Operative Venous Thromboembolismin Asian Countries. Int J Angiol 2004;13:101-8.

38. Januel JM, Chen G, Ruffieux C, et al. Symptomatic inhospital deep vein thrombosis and pulmonary embolism following hip and knee arthroplasty among patients receiving recommended prophylaxis: a systematic review. JAMA 2012;307:294-303.

39. Zeng Y, Si H, Wu Y, et al. The incidence of symptomatic in-hospital VTEs in Asian patients undergoing joint arthroplasty was low: a prospective, multicenter, 17,660-patient-enrolled cohort study. Knee Surg Sports Traumatol Arthrosc 2019;27:1075-82.

40. Forster R, Stewart M. Anticoagulants (extended duration) for prevention of venous thromboembolism following total hip or knee replacement or hip fracture repair. Cochrane Database Syst Rev 2016;3:CD004179.

41. McDougall CJ, Gray HS, Simpson PM, et al. Complications related to therapeutic anticoagulation in total hip arthroplasty. J Arthroplasty 2013;28:187-92.

42. Kim YT, Kang MW, Lee JK, et al. Combined use of topical intraarticular tranexamic acid and rivaroxaban in total knee arthroplasty safely reduces blood loss, transfusion rates, and wound complications without increasing the risk of thrombosis. BMC Musculoskelet Disord 2018;19:227. 